\title{
Thrombolysis during out-of-hospital cardiac arrest: a lesson in the law of diminishing returns
}

\author{
James M Dargin' and Lillian L Emlet ${ }^{2}$ \\ University of Pittsburgh Department of Critical Care Medicine: Evidence-Based Medicine Journal Club, edited by Eric B Milbrandt
}

\begin{abstract}
Expanded Abstract
Citation

Böttiger BW, Arntz HR, Chamberlain DA, Bluhmki E, Belmans A, Danays T, Carli PA, Adgey JA, Bode C, Wenzel V: Thrombolysis during resuscitation for out-of-hospital cardiac arrest. N Engl J Med 2008, 359:2651-2662 [1].
\end{abstract}

\section{Background}

Approximately $70 \%$ of persons who have an out-of-hospital cardiac arrest have underlying acute myocardial infarction or pulmonary embolism. Therefore, thrombolysis during cardiopulmonary resuscitation may improve survival.

\section{Methods}

Objective: To determine whether thrombolysis with the use of tenecteplase during cardiopulmonary resuscitation can improve survival in adults with witnessed out-of-hospital arrest of presumed cardiac origin.

Design: Prospective, randomized, double-blind, placebo-controlled, multicenter trial.

Setting: 66 European emergency medical-service systems.

Subjects: 1050 adult patients with witnessed out-of-hospital cardiac arrest.

Intervention: We randomly assigned adult patients with witnessed out-of-hospital cardiac arrest to receive tenecteplase or placebo during cardiopulmonary resuscitation. Adjunctive heparin or aspirin was not used.

Outcomes: The primary end point was 30-day survival; the secondary end points were hospital admission, return of spontaneous circulation, 24-hour survival, survival to hospital discharge, and neurologic outcome.

\section{Results}

After blinded review of data from the first 443 patients, the data and safety monitoring board recommended discontinuation of enrollment of asystolic patients because of low survival, and the protocol was amended. Subsequently, the trial was terminated prematurely for futility after enrolling a total of 1050 patients. Tenecteplase was administered to 525 patients and placebo to 525 patients; the two treatment groups had similar clinical profiles. We did not detect any significant differences between tenecteplase and placebo in the primary end point of 30-day survival ( $14.7 \%$ vs. $17.0 \%$; $P=0.36$; relative risk, $0.87 ; 95 \%$ confidence interval, 0.65 to 1.15 ) or in the secondary end points of hospital admission ( $53.5 \%$ vs. $55.0 \%, P=0.67$ ), return of spontaneous circulation ( $55.0 \%$ vs. $54.6 \%, P=0.96$ ), 24-hour survival (30.6\% vs. 33.3\%, $P=0.39$ ), survival to hospital discharge (15.1\% vs. 17.5\%, $P=0.33$ ), or neurologic outcome $(\mathrm{P}=0.69)$. There were more intracranial hemorrhages in the tenecteplase group.

\section{Conclusions}

When tenecteplase was used without adjunctive antithrombotic therapy during advanced life support for out-ofhospital cardiac arrest, we did not detect an improvement in outcome, in comparison with placebo. (ClinicalTrials.gov number, NCT00157261.)

\section{Commentary}

Intravascular thrombosis plays a fundamental role in the pathophysiology of cardiac arrest. Autopsy results from

*Correspondence: darginjm@Upmc.edu

'Department of Critical Care Medicine, University of Pittsburgh School of Medicine, Pittsburgh, Pennsylvania, USA

Full list of author information is available at the end of the article cases of unsuccessful resuscitation and coronary angiography in survivors of out-of-hospital cardiac arrest suggest that $50-70 \%$ of deaths can be attributed to thrombosis in the form of myocardial infarction or pulmonary embolism [2,3]. Ischemia and reperfusion during resuscitation from cardiac arrest cause endothelial cell dysfunction, platelet activation, disseminated intravascular coagulation, relatively low fibrinolysis, and a propensity for microcirculatory clot formation $[4,5]$. 
Microcirculatory thrombosis leading to a "no-reflow" phenomenon after return of spontaneous circulation may contribute to poor neurological function after cardiac arrest $[6,7]$. A number of studies have evaluated the efficacy of thrombolysis during out-of-hospital cardiopulmonary resuscitation. A meta-analysis of these studies, including one prospective and seven retrospective studies, demonstrated an improvement in return of spontaneous circulation, survival to admission, 24-hour survival, hospital discharge, and neurological outcome [8]. Based on these results, the authors concluded that a large, randomized, multicenter study should be conducted to determine the efficacy of thrombolysis during cardiac arrest.

The Thrombolysis in Cardiac Arrest (TROICA) trial investigators conducted a prospective double-blind, randomized, placebo-controlled trial in 66 European emergency medical-service systems (EMS) [1]. Adults with witnessed out-of-hospital cardiac arrest with an EMS response time of less than ten minutes were eligible for the study. The study protocol permitted open-label use of thrombolytics rather than randomization for cases in which pulmonary embolism was suspected as the cause of arrest. Patients with an initial rhythm of asystole or pulseless electrical activity were immediately randomized to weight-based tenecteplase or placebo, and patients with ventricular fibrillation or pulseless ventricular tachycardia were randomized after three failed attempts at defibrillation. Adjunctive antithrombotic and antiplatelet agents were not administered. The trial was suspended after futility analyses were performed on data from 653 patients. A total of 1050 patients were enrolled and no patient was lost to 30-day follow-up. The baseline characteristics of the two groups were well matched in terms of age, comorbidities, and long-term medications, including aspirin and warfarin. EMS response times were similar between groups and median time to study drug administration was 18 minutes. The circumstances of cardiac arrest were similar between groups, including the initial rhythm, cardiopulmonary resuscitation (CPR) by bystanders, and defibrillation administered by first responder. There was no difference between tenecteplase and placebo in the primary endpoint of 30-day survival or for any of the secondary endpoints, though there was a higher rate of intracranial hemorrhage in the tenecteplase group. The authors concluded that tenecteplase without an adjunctive antithrombotic during CPR does not improve outcome for out-of-hospital cardiac arrest.

The TROICA trial has several strengths, including the large sample size, multicenter design, evaluation of clinically important outcomes, and complete follow-up for the primary endpoint. Of particular note is the time to thrombolysis of 18 minutes from collapse, which represents a significantly shorter time than the typical 30 minutes cited in previous studies. Despite these strengths, the study is subject to a few important limitations. Most detailled information regarding inhospital care was lacking, which may have affected the primary outcome of 30-day survival. In addition, survival data may be subject to selection bias as the authors allowed - for ethical reasons - the open-label use of thrombolytics for suspected pulmonary embolism, potentially excluding from randomization a subgroup of patients likely to benefit from thrombolysis. Despite these limitations, the TROICA Trial convincingly demonstrates no mortality benefit from thrombolysis with tenecteplase and an increase risk of asymptomatic intracranial hemorrhage in patients with out-of-hospital cardiac arrest.

The search for new interventions to improve outcomes for out-of-hospital cardiac arrest remains elusive. Why did the current trial fail to show a benefit for thrombolysis despite a strong biologic rationale and a suggestion of benefit in prior, albeit smaller, studies? Decreased perfusion pressure may have prevented drug delivery and reduced the efficacy of thrombolytics. Alternatively, the negative result seen in the TROICA trial could be ascribed to a lack of adjunctive antithrombotic or antiplatelet agents, given that all eight studies in the Li et al meta-analysis used heparin with or without aspirin [8]. The most likely explanation, however, may be the law of diminishing returns. The TROICA trial was conducted within a well-optimized EMS system, as evidenced by the rapid EMS response and time to thrombolysis. Furthermore, the authors selected a patient population with potential for a favorable outcome, as evidenced by the 30 -day survival of $17 \%$ in the placebo group compared to $10 \%$ in most studies [9]. The corollary to this is that the incremental benefit of pre-hospital advanced life support beyond early CPR and defibrillation tends to be minimal, a lesson learned from The Ontario Prehospital Advanced Life Support (OPALS) study [10].

\section{Recommendation}

Based on the results of the TROICA trial, there seems to be no benefit from the use of tenecteplase without adjunctive antithrombotic therapy in out-of-hospital cardiac arrest. No such conclusion can be made regarding the subgroup of patients with suspected pulmonary embolism and the results should not be generalized to the inpatient setting.

\section{Competing interests}

The authors declare that they have no competing interests.

\section{Author details}

${ }^{1}$ Clinical Fellow, Department of Critical Care Medicine, University of Pittsburgh School of Medicine, Pittsburgh, Pennsylvania, USA. ${ }^{2}$ Assistant Professor, Department of Critical Care Medicine, University of Pittsburgh School of Medicine, Pittsburgh, Pennsylvania, USA

Published: 22 March 2010 


\section{References}

1. Böttiger BW, Arntz HR, Chamberlain DA, Bluhmki E, Belmans A, Danays T, Carli PA, Adgey JA, Bode C, Wenzel V: Thrombolysis during resuscitation for out-of-hospital cardiac arrest. N Engl J Med 2008, 359:2651-2662.

2. Silfvast T: Cause of death in unsuccessful prehospital resuscitation. J Intern Med 1991, 229:331-335.

3. Spaulding CM, Joly LM, Rosenberg A, Monchi M, Weber SN, Dhainaut JF, Carli P: Immediate coronary angiography in survivors of out-of-hospital cardiac arrest. N Engl J Med 1997, 336:1629-1633.

4. Böttiger BW, Motsch J, Böhrer H, Böker T, Aulmann M, Nawroth PP, Martin E: Activation of blood coagulation after cardiac arrest is not balanced adequately by activation of endogenous fibrinolysis. Circulation 1995 , 92:2572-2578.

5. Böttiger BW, Böhrer H, Böker T, Motsch J, Aulmann M, Martin E: Platelet factor 4 release in patients undergoing cardiopulmonary resuscitation-can reperfusion be impaired by platelet activation? Acta Anaesthesio/ Scand 1996, 40:631-635.

6. Fischer M, Böttiger BW, Popov-Cenic S, Hossmann KA: Thrombolysis using plasminogen activator and heparin reduces cerebral no-reflow after resuscitation from cardiac arrest: an experimental study in the cat. Intensive Care Med 1996, 22:1214-1223.
7. Lederer W, Lichtenberger C, Pechlaner C, Kinzl J, Kroesen G, Baubin M: Longterm survival and neurological outcome of patients who received recombinant tissue plasminogen activator during out-of-hospital cardiac arrest. Resuscitation 2004, 61:123-129.

8. Li X, Fu QL, Jing XL, Li Y, Zhan H, Ma ZF, Liao XX: A meta-analysis of cardiopulmonary resuscitation with and without the administration of thrombolytic agents. Resuscitation 2006, 70:31-36.

9. Rea TD, Eisenberg MS, Sinibaldi G, White RD: Incidence of EMS-treated outof-hospital cardiac arrest in the United States. Resuscitation 2004, 63:17-24.

10. Stiell IG, Wells GA, Field B, Spaite DW, Nesbitt LP, De Maio VJ, Nichol G, Cousineau D, Blackburn J, Munkley D, Luinstra-Toohey L, Campeau T, Dagnone E, Lyver M: Advanced cardiac life support in out-of-hospital cardiac arrest. N Engl J Med 2004, 351:647-656.

doi:10.1186/cc8906

Cite this article as: Dargin JM, Emlet LL: Thrombolysis during out-ofhospital cardiac arrest: a lesson in the law of diminishing returns. Critical Care 2010, 14:304. 